\title{
Generating the Curvature Perturbation at the End of Inflation in String Theory
}

\author{
David H. Lyth ${ }^{1}$ and Antonio Riotto ${ }^{2}$ \\ ${ }^{1}$ Physics Department, Lancaster University, Lancaster LA1 4YB, United Kingdom \\ ${ }^{2}$ CERN, Theory Division, Geneve 23, CH-1211 Switzerland, \\ and INFN, Sezione di Padova, Via Marzolo 8, 35131, Italy
}

(Received 17 July 2006; published 18 September 2006)

\begin{abstract}
In brane inflationary scenarios, the cosmological perturbations are supposed to originate from the vacuum fluctuations of the inflaton field corresponding to the position of the brane. We show that a significant, and possibly dominant, contribution to the curvature perturbation is generated at the end of inflation through the vacuum fluctuations of fields, other than the inflaton, which are light during the inflationary trajectory and become heavy at the brane-antibrane annihilation. These fields appear generically in string compactifications where the background geometry has exact or approximate isometries and parametrize the internal angular directions of the brane.
\end{abstract}

PACS numbers: $98.80 . \mathrm{Cq}, 11.25 . \mathrm{Wx}$

One of the basic ideas of modern cosmology is that there was an epoch early in the history of the Universe when a potential, or vacuum energy, dominated other forms of energy densities such as matter or radiation. During this epoch, dubbed inflation, the scale factor grew quasiexponentially in time $[1,2]$. Inflation has become the dominant paradigm for understanding the homogeneity and isotropy of our observed Universe and the initial conditions for structure formation and for cosmic microwave background (CMB) anisotropy. In the inflationary picture, primordial density and gravity-wave fluctuations are created from quantum fluctuations "redshifted" out of the horizon during the early period of superluminal expansion of the Universe, where they are "frozen" [3,4]. Perturbations at the surface of last scattering are observable as temperature anisotropy in the $\mathrm{CMB}$, which was first detected by the Cosmic Background Explorer satellite [5,6]. The last and most impressive confirmation of the inflationary paradigm has been recently provided by the three-year data of the Wilkinson Microwave Anistropy Probe (WMAP) mission, which has marked the beginning of the precision era of the CMB measurements in space [7]. The WMAP Collaboration has produced a full-sky map of the angular variations of the CMB, with unprecedented accuracy. WMAP data confirm the inflationary mechanism as responsible for the generation of curvature (adiabatic) superhorizon fluctuations.

In the standard scenario, inflation is driven by the potential energy associated to a scalar field, the inflaton, whose potential is flat enough to support a sufficiently long period of acceleration. Despite the simplicity of the inflationary paradigm, it is not yet clear which high energy particle physics setup can most naturally incorporate cosmological inflation [2]. On the other hand, since a consistent and renormalizable treatment of the gravitational interactions leads to string theory, it is natural to ask whether a primordial period of inflation may be predicted within a stringy setup, possibly making use of the extra spatial dimensions and of nonperturbative objects ( $D$-branes) whose presence is predicted by string theory.

Significant progress has been recently made along these lines [8-10] within specific string theory constructions, giving rise to the idea of brane inflation. In these string constructions, a $D$-brane and an anti- $D$-brane live in extra spatial dimensions and their distance plays the role of the inflaton field in the four-dimensional effective theory. The potential between the $D$-brane and an anti- $D$-brane may be sufficiently flat to support a prolonged period of inflation. Furthermore, if the geometry of the extra dimensions is warped, inflation may be obtained even more easily, since the warped geometry redshifts the brane tension and reduces (usually by a large factor) the mutual attraction between the branes and antibranes. Therefore, warped throats provide an efficient way to achieve brane inflation. In this respect, the concept of flux compactification has become an ideal setup to realize brane inflation. Stable fluxes of higher-dimensional fields may be present in the extra dimensions and provide a promising mechanism to stabilize the moduli fields [11-13], such as field parametrizing the overall volume of the extra dimensions, giving them heavy masses and, therefore, a consistent string theory. Furthermore, flux compactifications generically induce warped spaces, thus providing a nice setup for inflation.

In the Kachru-Kallosh-Linde-Maldacena-McAllisterTrivedi (KKLMMT) scenario [14,15], for instance, the entire physics of inflation takes place in the throat region, and it is mostly insensitive to the details of the ultraviolet Calabi-Yau region. In the KKLMMT scenario, an anti-D3-brane is sitting at the infrared end of the throat and a $D 3$-brane is moving toward it. In the absence of antibranes, the $D 3$ is a supersymmetric object and it feels no force [16]. The only source of supersymmetry breaking in the compactification is the inclusion of the anti-D3-brane. The insertion of a distant anti-D3-brane 
just produces a very flat potential for the scalar field corresponding to the position of the D3-brane, which plays the role of the inflaton field. Inflation ends when the branes come close together and annihilate, allowing the Universe to settle down to the string vacuum state that describes our Universe.

Despite the simplicity of the brane inflationary scenario, the mechanism by which cosmological adiabatic perturbations are generated is not yet established. In the common lore, the curvature perturbation $\zeta$ is supposed to originate from the vacuum fluctuations of the inflaton field itself. In this Letter, we explore a different possibility, that a significant, and possibly dominant, contribution to the curvature perturbation is generated at the end of inflation through the vacuum fluctuations of fields, other than the inflaton, which are light during the inflationary trajectory and become heavy at the brane-antibrane annihilation. Indeed, if the branes are embedded in a string compactification where the background geometry has exact or approximate isometries, there are scalar brane degrees of freedom transverse to the mutual distance and parametrizing the internal angular directions of the mobile brane. Their potential is so flat that they have practically no influence on the inflaton trajectory. However, being almost massless during the de Sitter epoch, their quantum fluctuations are excited with a nearly scale-invariant spectrum. The generation of the curvature perturbation in such a case is due to the fact that the end of inflation happens in different space points at different instants of time. More specifically, inflation ends when the mobile brane and the antibrane come close together and annihilate. At each position in space, inflation ends when the inflaton field $\phi$ has some critical value $\phi_{e}$. Usually, it is assumed that the end of inflation is controlled entirely by the inflaton, so that $\phi_{e}$ is independent of the position in space. Then inflation ends on a spacetime slice of uniform energy density. However, the value $\phi_{e}$ depends on the internal angular directions of the branes and, if these phases are quantum mechanically excited during inflation, the value $\phi_{e}$ will depend upon the position in space.

Under these circumstances, there is no reason why inflation should end on a slice of uniform energy density, and one expects the comoving curvature perturbation $\zeta_{e}$ to be generated at the end of inflation [17-20] when the light fields become very massive and decay (after the end of the inflation, the branes have disappeared and certainly cannot fluctuate anymore) promptly even if they are gravitationally coupled to ordinary matter.

Since the mechanism described in this Letter originates from the isometries of the compactification geometry where the mobile branes move, we expect it to be rather common in inflationary models built up within stringy setups.

As a specific and concrete example, let us discuss again the KKLMMT model for $D$-brane inflation $[21,22]$. The KKLMMT scenario corresponds to a Calabi-Yau geometry that develops a long warped anti-de Sitter (AdS)-like throat, and it corresponds to a compactification of the Klebanov-Strassler (KS) solution [16]. The KS solution has a $S U(2) \otimes S U(2)$ isometry group. For example, the ultraviolet geometry of the throat is that of a cone over the Einstein manifold $T^{1,1}=S U(2) \otimes S U(2) / U(1)$. In the infrared region, the singular tip of the cone is smoothened out to a three-sphere, which still preserves a $S U(2) \otimes$ $S U(2)$ symmetry.

During its motion toward the infrared, the D3-brane can fluctuate in the internal angular directions. Since during the inflationary period the two brane setup approximately preserves the $S U(2) \otimes S U(2)$ symmetry, the scalar fields associated with the angular positions are almost massless and lighter than the Hubble rate. Furthermore, being angles, they do not get masses from the volume stabilization mechanism. In the Kähler potential of four-dimensional supergravity, the volume modulus $\rho$ always appears in the combination $\rho+\bar{\rho}-K\left(\phi_{\alpha}, \bar{\phi}_{\alpha}\right)$ [23], where $\phi_{\alpha}$ collectively denote the position of the branes in the six internal directions, and $K$ is the Kähler potential for the geometry. This coupling generates a mass for the fluctuations $\phi_{\alpha}$. However, since the geometry has isometries, $K\left(\phi_{\alpha}, \bar{\phi}_{\alpha}\right)$ does not depend on some of the angles. In this way, the form of potential for the angular fluctuations is not affected by the stabilization mechanism and leaves some angles much lighter than the Hubble rate during inflation. At the end of inflation, the D3-brane comes close to the anti- $D 3$-brane. At stringy distances, we cannot ignore the fields coming from open strings connecting the branes. In particular, in a generic brane-antibrane system, the open string ground state is a tachyon $T$ with mass of order $M_{s}$, the string scale.

Since the annihilation takes place at the bottom of the throat, the tachyonic mass is redshifted to $m_{T}^{2} \sim-a_{0}^{2} M_{s}^{2}$, where $a_{0}$ is the redshift factor at the bottom of the throat. Unfortunately, the precise potential is rather dependent on the detailed structures of the compactification and remains to be explored more carefully. However, to illustrate our point, we can write a potential for the inflaton $\phi$, the tachyon $T$, and the angular positions $\theta_{\alpha}$ which captures the salient features during inflation and of the tachyonic condensation when the brane and antibrane are close to each other. Keeping for illustrative purposes just one angular position, we write

$$
\begin{aligned}
V(\phi, T, \theta)= & \frac{m^{2}}{2} \phi^{2}+T_{3} a_{0}^{4}\left[1-c\left(\frac{a_{0} M_{s}}{\phi}\right)^{4}\right] \\
& +T^{2}\left[\phi^{2}-a_{0}^{2} M_{s}^{2}(a+b \cos \theta)\right]+\cdots,
\end{aligned}
$$

where $T_{3}$ is the tension of a $D 3$-brane and the ellipses account for higher-order power terms in the field $T$ and of possible terms responsible for tiny masses for the phase $\theta$. One expects $a \sim b \sim 1$, and the coefficient $c=$ $(1 / M K) \ll 1$, where $M$ and $K$ are large integers parametrizing the flux units. 
The second line vanishes during inflation and specifies the inflaton field value $\phi_{e}$ at the end:

$$
\phi_{e}^{2}=a_{0}^{2} M_{s}^{2}(a+b \cos \theta) .
$$

The quadratic term for the inflaton field $\phi$ receives contributions from a number of sources and is rather modeldependent $[14,24]$. However, $m^{2}$ is expected to be comparable to $H^{2}=V_{0} / 3 M_{p}^{2}$, where $M_{p}$ is the reduced Planck mass $\left(G^{-1}=8 \pi M_{p}^{2}\right)$ (this might represent an obstacle in getting a sufficient number of $e$-foldings, but various ways to solve this problem have been proposed [25]).

As long as $\phi$ is much larger than the (redshifted) string scale, $T$ has a large positive mass, its vacuum expectation value $\langle T\rangle$ vanishes, and all the terms in the potential involving $T$ can be ignored. The inflationary potential is then independent of the angle $\theta$. At stringy distances, $T$ becomes tachyonic and triggers the brane-antibrane annihilation. When $T$ condenses, all the fluctuation fields on the branes acquire masses roughly of order of the redshifted string scale. These masses are $\mathcal{O}\left(a_{0} M_{s}\right)$ and much larger than the value of the Hubble rate $H \sim a_{0}^{2} M_{s}$ at annihilation. All of these massive fields will eventually decay.

There are two effects that break the $S U(2) \otimes S U(2)$ invariance and may prevent the angular fluctuations of the brane from being exactly massless during inflation [21].

There is a small source of breaking of the $S U(2) \otimes$ $S U(2)$ symmetry due to the presence of the anti- $D 3$-brane in the infrared, which sits at a specific point on the three-sphere. This explicit breaking is suppressed, though, when the distance between the two branes during the inflationary period is sizable. The second effect is caused by the moduli stabilization that requires fluxes in the ultraviolet region typically breaking $S U(2) \otimes S U(2)$; it is well known indeed that a Calabi-Yau manifold has no isometries at all. Even this second effect is suppressed by the distance. An estimate shows that the typical mass induced by the $S U(2) \otimes S U(2)$ breaking scales as $a^{8}(\phi)$ [26], where $a(\phi)$ is the warp factor evaluated at the position $\phi$ of the brane. This mass contribution is, therefore, suppressed compared to the Hubble rate during inflation.

There is a dual interpretation of these massless fields. As familiar from the AdS/conformal field theories correspondence and the holographic interpretation, the throat part of the compactification can be effectively replaced by a strongly interacting gauge theory coupled to the fourdimensional gravity. In this picture, the D3-brane position corresponds to a flat direction in the moduli space of vacua of the gauge theory. The global symmetry $S U(2) \otimes S U(2)$ is spontaneously broken along this flat direction [27]. The angular positions of the brane are, therefore, identified with the massless Goldstone bosons of the spontaneously broken global symmetry.

Now we turn to the calculation of the curvature perturbation. The phase will be $\theta \simeq \sigma / \phi_{e}$, where $\sigma$ is canoni- cally normalized. Using the $\delta N$ formalism, we may compute the spectrum of the curvature perturbation $[19,20]$

$$
\begin{gathered}
\mathcal{P}_{\zeta}=\left[\left(\frac{\partial N}{\partial \phi}\right)^{2}+\left(\frac{\partial N}{\partial \sigma}\right)^{2}\right]\left(\frac{H}{2 \pi}\right)^{2}, \\
\left(\frac{\partial N}{\partial \phi}\right)^{2}=\left.\frac{1}{M_{p}^{2}} \frac{V}{V^{\prime}}\right|_{*} \\
\left(\frac{\partial N}{\partial \sigma}\right)^{2}=\left.\frac{1}{M_{p}^{2}} \frac{V}{V^{\prime}}\right|_{e}\left(\frac{d \phi_{e}}{d \sigma}\right)^{2} .
\end{gathered}
$$

In Eq. (3), the first piece corresponds to the curvature perturbation generated during inflation, while the second to the curvature perturbation $\zeta_{e}$ generated at the end of inflation. It coincides with the perturbation of the change of the number of $e$-folds from a spacetime slice of uniform energy density just before the end of inflation to a spacetime slice of uniform energy density just after the end of inflation. In Eqs. (4) and (5), $V(\phi)$ is the potential during inflation and $3 M_{p}^{2} H^{2}=V \simeq T_{3} a_{0}^{4}$. The asterisk denotes the epoch when cosmological scales leave the horizon, and the subscript $e$ denotes the end of inflation. For a typical value of $\theta$, we have $\phi_{e} \sim a_{0} M_{s}$ and $d \phi_{e} / d \sigma \sim 1$.

The first contribution is generated at the horizon exit by the fluctuation of the inflaton, and the second contribution is generated at the end of inflation by the fluctuation of the phase field. (We assume that $\zeta$ remains constant afterward.) Let us see if the latter contribution can dominate. This will not happen if the mass term is negligible, because then $V^{\prime} / V \propto \phi^{-5}$ is increasing with time.

Suppose, instead, that the mass term dominates throughout inflation. This is the case if

$$
c \ll \eta \phi_{e}^{2} / M_{p}^{2},
$$

where $\eta \equiv m^{2} / 3 H_{*}^{2}$. Then we have $V^{\prime} / V \propto \phi$ and

$$
\phi_{*}=e^{N \eta} \phi_{e} .
$$

We are now dealing with the model investigated in Ref. [20], using a somewhat different notation. For typical values of $\theta$, the fluctuation of the phase field dominates if $\eta N \gtrsim 1$. Then the spectrum has the observed value if

$$
5 \times 10^{-4} \sim \frac{\sqrt{T_{3}} a_{0}}{\eta M_{s} M_{p}},
$$

which can be achieved by a suitable choice of parameters.

As $c$ is increased with the other parameters fixed, there is a continuous transition to the unviable case that the mass term is negligible throughout inflation. This transition is complete when $c \sim \eta \phi_{e}^{2} \sqrt{\eta N} / M_{p}^{2}$.

The spectral index of the curvature perturbation is determined by the potential of $\sigma$ evaluated at the horizon exit through the expression $[2,28] n=1+2 V^{\prime \prime}(\sigma) / 3 H^{2}$. The potential is roughly $m_{\sigma}^{2} \phi_{*}^{2} \cos \left(\sigma / \phi_{*}\right)$, and, if $m_{\sigma}^{2} / H^{2}$ is tiny as we suggested earlier, $n$ is indistinguishable from 1 , 
which is consistent with observation at the $2-3-\sigma$ level [29].

Finally, we consider non-Gaussianity with the mass term dominating. Assuming that the fluctuation of the phase field dominates, the parameter for the primordial nonGaussianity is

$$
\frac{3}{5} f_{\mathrm{NL}}=\frac{\partial^{2} N}{\partial \sigma^{2}}\left(\frac{\partial N}{\partial \sigma}\right)^{-2} \sim \eta \frac{\phi_{e}}{M_{p}} \frac{d^{2} \phi_{e}}{d \sigma^{2}}\left(\frac{d \phi_{e}}{d \sigma}\right)^{-2} .
$$

We need $\left|f_{\mathrm{NL}}\right| \gtrsim 1$ for it to be eventually observable [30]. As was investigated in Ref. [20], that may be possible for a very special choice of the phase angle, but there seems to be no reason to make such a choice. In particular, anthropic selection does not seem to favor (or disfavor) significant non-Gaussianity [31].

To summarize, we have pointed out that, in inflationary scenarios built up in string models, where inflation is driven by mobile branes moving in certain compactification manifolds, the cosmological perturbations may be generated at the final stage of inflation, when the brane and antibrane annihilate, through the vacuum fluctuations of pseudo-Nambu-Goldstone fields. They are associated to the isometries present when the brane and antibrane are far apart and disappear close to the tachyonic point. The generation of the curvature perturbation in such a case is due to the fact that the end of inflation happens in different space points at different instants of time. As a specific example, we have discussed the KKLMMT scenario, but we expect our findings to be quite generic. Furthermore, even though for illustrative purposes we have analyzed the case of only one flat direction, in the realistic case there are likely to be many flat directions and many NambuGoldstone bosons. This, in principle, opens up the possibility that some isocurvature component(s) might be produced in addition to the adiabatic one. However, we expect all of the Nambu-Goldstone bosons to promptly decay into radiation at the brane and antibrane annihilation and, therefore, no residual isocurvature perturbation to survive.

[1] A. Guth, Phys. Rev. D 23, 347 (1981).

[2] D. H. Lyth and A. Riotto, Phys. Rep. 314, 1 (1999); A. Riotto, hep-ph/0210162; W. H. Kinney, astro-ph/0301448.

[3] V.F. Mukhanov and G. V. Chibisov, JETP Lett. 33, 532 (1981).

[4] J. M. Bardeen, P. J. Steinhardt, and M. S. Turner, Phys. Rev. D 28, 679 (1983).

[5] C. L. Bennett et al., Astrophys. J. 464, L1 (1996).

[6] K. M. Gorski et al., Astrophys. J. 464, L11 (1996).

[7] D. N. Spergel et al. (WMAP Collaboration), Astrophys. J. Suppl. Ser. 148, 175 (2003).

[8] G. R. Dvali and S.-H.H. Tye, Phys. Lett. B 450, 72 (1999).

[9] C. P. Burgess, M. Majumdar, D. Nolte, F. Quevedo, G. Rajesh, and R. J. Zhang, J. High Energy Phys. 07 (2001) 047.

[10] G. R. Dvali, Q. Shafi, and S. Solganik, hep-th/0105203.
[11] S. B. Giddings, S. Kachru, and J. Polchinski, Phys. Rev. D 66, 106006 (2002).

[12] S. Kachru, R. Kallosh, A. Linde, and S. P. Trivedi, Phys. Rev. D 68, 046005 (2003).

[13] M. Grana, Phys. Rep. 423, 91 (2006).

[14] S. Kachru, R. Kallosh, A. Linde, J. Maldacena, L. McAllister, and S. P. Trivedi, J. Cosmol. Astropart. Phys. 10 (2003) 013.

[15] H. Firouzjahi and S.-H.H. Tye, J. Cosmol. Astropart. Phys. 03 (2005) 009.

[16] I. Klebanov and M. J. Strassler, J. High Energy Phys. 08 (2000) 052.

[17] F. Bernardeau and J. P. Uzan, Phys. Rev. D 67, 121301 (2003).

[18] F. Bernardeau, L. Kofman, and J. P. Uzan, Phys. Rev. D 70, 083004 (2004).

[19] D. H. Lyth, J. Cosmol. Astropart. Phys. 11 (2005) 006; M. P. Salem, Phys. Rev. D 72, 123516 (2005).

[20] L. Alabidi and D. Lyth, astro-ph/0604569.

[21] This example was first mentioned in L. Pilo, A. Riotto, and A. Zaffaroni, J. High Energy Phys. 07 (2004) 052; G. F. Giudice, A. Riotto, and A. Zaffaroni, Nucl. Phys. B710, 511 (2005).

[22] L. Kofman, in Proceedings of the 22nd IAP Colloquium, Paris, 2006 (unpublished).

[23] O. DeWolfe and S. B. Giddings, Phys. Rev. D 67, 066008 (2003).

[24] S. E. Shandera, J. Cosmol. Astropart. Phys. 04 (2005) 011.

[25] J.P. Hsu, R. Kallosh, and S. Prokushkin, J. Cosmol. Astropart. Phys. 12 (2003) 009; F. Koyama, Y. Tachikawa, and T. Watari, Phys. Rev. D 69, 106001 (2004); J. P. Hsu and R. Kallosh, J. High Energy Phys. 04 (2004) 042; C. P. Burgess, J. M. Cline, H. Stoica, and F. Quevedo, J. High Energy Phys. 09 (2004) 033; N. Iizuka and S. P. Trivedi, Phys. Rev. D 70, 043519 (2004).

[26] O. DeWolfe, S. Kachru, and H. Verlinde, J. High Energy Phys. 05 (2004) 017.

[27] The setup with $K$ and $M$ fluxes is dual to a $S U(K M+$ $M) \otimes S U(K M) N=1$ supersymmetric theory with pairs of bifundamental fields $A_{i}$ and $B_{i}, i=1,2$, each one rotated by a global $S U(2)$ symmetry, with a $S U(2) \otimes$ $S U(2)$ invariant superpotential. The same compactification with a $D 3$-brane inserted can be represented by a gauge group $S U(K M+M+1) \otimes S U(K M+1)$ broken to $S U(K M+M) \otimes S U(K M)$ along a flat direction where $A, B$ acquire a vacuum expectation value breaking the global symmetry $S U(2) \otimes S U(2)$.

[28] M. Sasaki and E. D. Stewart, Prog. Theor. Phys. 95, 71 (1996).

[29] D. N. Spergel et al., astro-ph/0603449; L. Alabidi and D. H. Lyth, astro-ph/0603539; W. H. Kinney, E. W. Kolb, A. Melchiorri, and A. Riotto, Phys. Rev. D 74, 023502 (2006).

[30] N. Bartolo, E. Komatsu, S. Matarrese, and A. Riotto, Phys. Rep. 402, 103 (2004); M. Liguori, F. K. Hansen, E. Komatsu, S. Matarrese, and A. Riotto, Phys. Rev. D 73, 043505 (2006); N. Bartolo, S. Matarrese, and A. Riotto, J. Cosmol. Astropart. Phys. 05 (2006) 010; 06 (2006) 024.

[31] D. H. Lyth, J. Cosmol. Astropart. Phys. 06 (2006) 015. 